\title{
Identical Quantum Particles as Distinguishable Objects
}

\author{
Dennis Dieks ${ }^{1} \cdot$ Andrea Lubberdink $^{1}$
}

Published online: 24 June 2020

(C) The Author(s) 2020

\begin{abstract}
According to classical physics particles are basic building blocks of the world. These classical particles are distinguishable objects, individuated by unique combinations of physical properties. By contrast, in quantum mechanics the received view is that particles of the same kind ("identical particles") are physically indistinguishable from each other and lack identity. This doctrine rests on the quantum mechanical (anti)symmetrization postulates together with the "factorist" assumption that each single particle is represented in exactly one factor space of the tensor product Hilbert space of a many-particle system. Even though standard in theoretical physics and the philosophy of physics, the assumption of factorism and the ensuing indistinguishability of particles are problematic. Particle indistinguishability is irreconcilable with the everyday meaning of "particle", and also with how this term is used in the practice of physics. Moreover, it is a consequence of the standard view that identical quantum particles remain indistinguishable even in the classical limit, which makes a smooth transition to the classical particle concept impossible. Lubberdink (1998; 2009) and Dieks and Lubberdink (2011) have proposed an alternative conception of quantum particles that does not rely on factorism and avoids these difficulties. We further explain and discuss this alternative framework here. One of its key consequences is that particles in quantum theory are not fundamental but emergent; another that once they have emerged, quantum particles are always physically distinguishable and thus possess a physically grounded identity.
\end{abstract}

Keywords Identical quantum particles $\cdot$ Distinguishability $\cdot$ Factorism $\cdot$ Fermions $\cdot$ Bosons

\section{Introduction}

The physical world of everyday experience consists of individual objects that can be distinguished from each other by their distinctive physical characteristics. In classical physics this picture is extended into the microscopic domain by the introduction of the notion of a

Andrea Lubberdink

andrealubberdink@gmail.com

Dennis Dieks

d.dieks@uu.nl

1 History and Philosophy of Science, Utrecht University, Buys Ballotgebouw, Princetonplein 5, 3584 CC Utrecht, The Netherlands 
particle: a classical particle is characterized by an individuating set of values of physical quantities (mass, electric charge, position in Newtonian absolute space, momentum, etc.). Although different classical particles may have a number of these values in common, there never is a complete overlap of particle properties because according to classical theory repulsive forces become increasingly strong (and eventually divergent) when mutual particle distances decrease, so that any two particles differ at least in their spatial locations.

Each classical particle accordingly is different from all the others: it possesses a physically defined identity, with which an identifying name or label can be associated. This labeling can take the form of providing definite descriptions that identify particles by explicitly specifying unique characteristics (e.g., "Call the particle in the bottom left corner of the container particle 1, the particle closest to it particle 2", etc.). Often, however, the possibility-in-principle of definite descriptions is taken for granted and the reference of the labels is left open as a matter of convention ("Consider a collection of particles and number them $1,2, \ldots, N ")$.

In classical physics there is therefore no need to invoke a notion of particle identity that goes beyond what can be based upon the physical characteristics specified by physical theories (values of physical quantities, relational properties, see also Sect. 4). This instantiates a general methodological principle, which we respect: In physics the use of metaphysical notions (in the sense of notions not connected to what can be defined in terms of standard physical quantities - in our case notions like "haecceity" or "primitive thisness") should be eschewed.

Which physical quantities are relevant for describing particles of a specific kind is governed by law-like physical principles (Lombardi and Dieks 2015). Generally speaking, two sorts of such quantities can be distinguished: quantities taking values that vary depending on the state of the particle (e.g., position and velocity), and state independent quantities that are typical of the kind of particle we are dealing with (e.g., electron rest mass, electron charge).

Quantum mechanics also describes elementary quantum systems by means of state dependent and state independent quantities. There is an important difference with classical mechanics, however: the state dependent quantities are now represented by operators and instead of the classical state (or "phase") spaces, vector spaces (Hilbert spaces) are used. Nevertheless there are similarities between the structures of quantum and classical mechanics: many functional relations between classical quantities are taken over in quantum mechanics as relations between operators, and where Poisson brackets between physical quantities occur in the formulas of classical theory, commutators between operators appear in corresponding quantum formulas.

It is important for the subject of this paper that there is also an analogy between classical and quantum mechanics with regard to how many-particle state spaces are built up from one-particle state spaces. Both classically and quantum mechanically, an $\mathrm{N}$-particles state space must be able to accommodate all kinematically possible combinations of oneparticle states. In classical physics, the state of one particle of a given kind is fixed by the values of a certain number, $n$ say, of (state dependent) physical quantities. Therefore we need the specification of $n \cdot N$ quantities to determine the total state of $N$ particles of the same kind; the $N$-particles state space must consequently be an $n \cdot N$-dimensional manifold of points (each point representing a possible state). If the one-particle state spaces are discrete, with $X$ possible states in each of them, the number of states in the $N$-particles state space thus becomes $X^{N}$. Similarly, in quantum mechanics an $N$-particle Hilbert space must be able to accommodate the possible combinations of one-particle states. However, the one-particle states are now defined as linear superpositions of a set of basis states (basis 
vectors) in a one-particle Hilbert space. If we assume, for the sake of illustration, that there is a finite number $X$ of such independent basis states (i.e., the one-particle Hilbert space is $X$-dimensional), the combination of all basis vectors of $N$ one-particle spaces leads to a set of $X^{N}$ new independent basis states, spanning the $X^{N}$ - dimensional "tensor" product space $\mathcal{H}^{N}=\mathcal{H}_{1} \otimes \mathcal{H}_{2} \otimes \mathcal{H}_{3} \otimes \cdots \otimes \mathcal{H}_{N}$

In this expression for the total Hilbert space, the one-particle Hilbert spaces (the factor spaces) are labeled, $1,2, \ldots, N$. This is analogous to the labeling of individual particle spaces, and the quantities defined in them, in the classical case. A point in an $N$-particles classical state space (representing $N$ particles of the same kind) has coordinates $\left(Z_{1}, Z_{2}, Z_{3}, \ldots, Z_{N}\right)$, where $Z_{i}$ denotes the set of quantities defining the state of particle $i$. These quantities are also the coordinates of the part of the total state space in which particle $i$ is represented. In this way, the total classical state space can be thought of as a combination of $N$ one-particle state spaces. Because each of these one-particle spaces represents one specific particle (singled out by its unique state and history), the labels $i$ can be used to label both the one-particle spaces and the particles themselves.

In view of the analogies between how the classical and quantum many-particle state spaces are built up it seems plausible to assume that also in the quantum case one-particle factor state spaces (occurring as factors in the total tensor product Hilbert space) and their labels are in one-to-one correspondence with the single particles in an $N$-particle system. This is in fact the standard view, both in physics and the philosophy of physics: factor space labels are associated with single particles, which allows us to speak of particle 1 , particle 2, and so forth. ${ }^{1}$

However, as we shall argue, there is in fact no valid parallelism between classical and quantum mechanics on this point: the factor space labels in the quantum mechanics of "identical particles" should not be thought of as referring to single particles. Our argument will crucially depend on a specific feature of the quantum formalism, namely that although the $\mathrm{N}$-particles state space possesses the structure of an $\mathrm{N}$-fold product of Hilbert spaces, the many-particle states in this space do not have the form of a concatenation of one-particle states, each in its own factor space. This complication is due to the symmetrization postulates, which ordain that states of many identical particles cannot be products of one-particle states.

\section{The Symmetrization Postulates}

Consider a product state $^{2}$ of the form $|L\rangle_{1}|R\rangle_{2}$, defined in a two-particles tensor product Hilbert space for particles of the same kind $\mathcal{H}_{1} \otimes \mathcal{H}_{2}$, with $|L\rangle$ and $|R\rangle$ standing for wave functions with support in narrow left and right regions of space, respectively. ${ }^{3}$ The natural

\footnotetext{
1 This standard doctrine- that factor space labels refer to single particles-has been baptized "factorism" by Caulton (Caulton 2014; Muller and Leegwater 2020). It constitutes the conceptual background of the "received view" concerning the nature of identical particles in quantum mechanics (French and Krause 2006).

${ }^{2}$ Note that we consider this state only for the sake of argument: As already mentioned, and as will be further explained in a moment, quantum mechanics does not allow the existence of product states for particles of the same kind.

3 The labels 1 and 2 are not strictly necessary here and in similar expressions: the factor spaces could be identified by their order, from left to right, in the expressions. The use of labels facilitates discussion, though.
} 
interpretation is that this state represents one particle located on the left and one on the right. This is supported by the fact that appropriate position measurements will with certainty result in one successful detection in the $L$ region and one in the $R$ region. The particles can in this (hypothetical!) case be individuated by the orthogonal states $|L\rangle$ and $|R\rangle$, and one may introduce physically meaningful particle labels in the same way as in classical physics. In fact, $L$ and $R$ themselves can function as labels.

Now reverse the order of the two particle states, so that the state $|R\rangle_{1}|L\rangle_{2}$ results. The particle that was originally represented in factor space 1 , namely particle $L$ (the left one) is now represented in factor space 2 , and the other particle is described in factor space $1 .{ }^{4}$ Now, given our principle that particles can only be individuated by physical quantities (in our case $L$ and $R$ ), the two states $|L\rangle_{1}|R\rangle_{2}$ and $|R\rangle_{1}|L\rangle_{2}$ represent the same physical situation: one particle on the left and one on the right. So we are facing a case of descriptional surplus structure. It is natural to remove the ensuing multiplicity of descriptions by defining one single representation: stipulate, by convention, that we call factor space 1 the one representing particle $L$, and similar for the particle $R$. We would thus obtain the unique representation $|L\rangle_{1}|R\rangle_{2}$.

This would be the same procedure as often followed in classical mechanics. As pointed out in the Introduction, there is a conventional choice to be made when we label classical particles, and this can be used to stipulate some unique ordering of one-particle states and their one-particle phase spaces. ${ }^{5}$ The multiplicity of theoretical descriptions in classical mechanics can thus be dealt with as a harmless consequence of the conventionality of labeling, ${ }^{6}$ and we may conventionally select one set of labels-e.g., by making use of definite descriptions-in order to remove the ambiguity. ${ }^{7}$

It is a very remarkable and important feature of quantum mechanics, however, that the multiplicity of physically equivalent states that we just discussed cannot occur at all. This is because it is decreed, by quantum law, that for systems of particles of the same kind product states like $|L\rangle_{1}|R\rangle_{2}$ and $|R\rangle_{1}|L\rangle_{2}$ are forbidden. Only a third type of state, namely an (anti)symmetric superposition of such product states, is allowed.

More precisely, there are two quantum postulates that govern the form of states of identical particles: a symmetrization postulate for bosonic systems, permitting only states that are invariant under permutations of the labels, and an antisymmetrization postulate for fermions (which introduces a minus sign for uneven permutations). Thus, instead of the product states $|L\rangle_{1}|R\rangle_{2}$ and $|R\rangle_{1}|L\rangle_{2}$, only one of the following two states is allowed:

\footnotetext{
4 This brings out a conceptual difference, in principle, between particle and factor space labels-a point that will be significant in our general analysis of the meaning of particle labels.

5 This is all based on the assumption that the particles are of the same kind. When two particles belong to different kinds, they have state independent physical properties that distinguish the individual state spaces. In that case swapping of states between such spaces, as considered here, will not be possible.

6 See Bigaj (2020) for an analysis of the meaning of label permutations that is somewhat different from the one given here.

7 A rarely seen alternative is to take the collection of states that relate to each other by label permutations as together representing the physical situation-this "super-state" has been called the Ehrenfest Z-star (Ehrenfest 1959 [1909]; Dieks and Lubberdink 2011). It bears some similarity to the quantum mechanical way of representing many-particle states, although the crucial quantum ingredient of superposition of states is of course lacking. Another, more common alternative for removing the multiplicity of labeling possibilities in classical mechanics is to go over to the reduced phase space, by identifying phase points that are mapped to each other by permutations of the labels.
} 


$$
|\Psi\rangle=\frac{1}{\sqrt{2}}\left\{|L\rangle_{1}|R\rangle_{2} \pm|R\rangle_{1}|L\rangle_{2}\right\}
$$

where the plus sign holds for the bosonic case and the minus sign applies to fermions.

As can be seen from the form of the total state in Eq. (1), the one-particle states $|L\rangle$ and $|R\rangle$ occur symmetrically in the factor spaces labeled by 1 and 2 , respectively. As a consequence, if in analogy with the classical case we are going to assume that these factor labels also refer to the single particles composing the total system, we have to conclude that the two particles are in exactly the same state. More generally, in the case of an (anti)symmetrical state of an $\mathrm{N}$-particle system all factor spaces contain the same one-particle states in precisely the same way so that the single-particle properties that are represented are identical. It is therefore impossible to individuate the "factorist particles" (i.e., particles labeled by the factor space indices) via different physical characteristics.

In a more formal way this conclusion can be reached by determining states associated with the individual factor spaces via the procedure of taking "partial traces": tracing out, in state (1), over the parts labeled by 2 we obtain the mixed state $W=1 / 2\{|L\rangle\langle L|+| R\rangle\langle R|\}$; and exactly the same state by tracing out over 1 . These two mixed states are standardly taken to be the one-particle quantum states defined in the single factor spaces 1 and 2, respectively; and these two states are identical. The conclusion generalizes to (anti)symmetric $N$-particle states, with $N>2$ : each factor space label is associated with the very same one-particle state.

\section{Problems of Factorism}

The correlation between one-particle state spaces and uniquely individuating particle states (one identifying state for each particle, represented in its unique one-particle state space), which in classical physics is the basis for the double use of factor space and particle labels, thus breaks down in quantum mechanics. If quantum particles are nevertheless taken to correspond to factor space labels, these particles must all be assigned exactly the same mixed one-particle state. Under the standard assumption that its quantum state exhausts what can be said about a physical system (i.e., the assumption that quantum mechanics is complete), it follows that all particles possess exactly the same physical properties. Although there have been discussions of this lack of differences in the literature, in particular in connection with the notion of "weak discernibility" (more about this in Sect. 4), we believe that it has not been sufficiently appreciated how strange, and adverse to the very idea of a particle, this consequence of factorism really is. On the contrary, it has become generally accepted both in theoretical physics and in the philosophy of physics literature that quantum particles are entities lacking individuality. It follows that a systematic formal description of quantum particles cannot use standard set theory but should resort to a variation on it that does without the notion of identity (French and Krause 2006; Krause and Arenhart 2019). ${ }^{8}$ Accordingly, it is possible to speak about the cardinality of a collection of particles (i.e., about how many particles are members of the collection), but it is not possible to pick out or address an individual particle.

\footnotetext{
${ }^{8}$ See also the recent restatement of this Received View in Krause and Arenhart (2019), and the references contained therein.
} 
The conceptual coherence of a scheme in which there are entities without individuality but nevertheless possessing a definite cardinality has recently been called into question by Bueno (2014) and Berto (2017). As Berto (2017) has forcefully argued, the notion of identity is essential for the very meaning of the concept of an entity, and we cannot have a collection of entities with a definite cardinality if these entities do not possess self-identities that make them different from each other. We agree with these a priori arguments. It follows from them that in order to be able to speak about $N$ particles at all, even if all their physical properties are the same, we have to allow identity markers, and labels, that are not physically grounded. ${ }^{9}$ What we want to draw attention to is the physical weirdness of the picture that thus results.

As the symmetrization postulates apply universally and globally to all particles of any given kind, for example all electrons in the universe, "factorists" must hold that each single electron is equally present at all positions in the universe at which there is "electron presence". For example, according to factorism it does not make sense to speak about the specific electrons in my body, since all electrons in the universe are equally (partly) present there. This result is not restricted to localization but holds in the same way for whatever physical particle property one may think of. All electrons share all their physical properties and are therefore mutually indistinguishable in all respects. The very same holds for all protons, neutrons and other particles of the same kind in the universe.

This leads to a lack of individuality that conflicts with the very notion of a physical particle, and is in stark contrast not only to how the notion of a particle functions in classical physics but also to how it is used in physical practice. This is important, because the very motivation for speaking about particles, also in the context of quantum mechanics, derives from analogies with classical physics and from the use that can be made of the particle concept in experimental practice.

To see a concrete illustration of the divide between the received view and physical practice, consider the Einstein-Podolsky-Rosen-Bohm state. In the foundational literature the EPR thought experiment (in its modern spin version due to Bohm) is standardly discussed as being about two electrons that find themselves at a large distance from each other and have a total spin state $\frac{1}{\sqrt{2}}\left\{|\uparrow\rangle_{1}|\downarrow\rangle_{2}-|\downarrow\rangle_{1}|\uparrow\rangle_{2}\right\}$. The spatial part of the wave function is often not written down explicitly, but it should be considered in order to make contact with the locality question that is at issue in the EPR discussion. The total state including its spatial part has the form

$$
|\Phi\rangle=\frac{1}{\sqrt{2}}\left\{|L\rangle_{1}|R\rangle_{2}+|R\rangle_{1}|L\rangle_{2}\right\} \otimes\left\{|\uparrow\rangle_{1}|\downarrow\rangle_{2}-|\downarrow\rangle_{1}|\uparrow\rangle_{2}\right\},
$$

where $|L\rangle$ and $|R\rangle$ as before are states localized on the left and right, respectively, at a large distance from each other. In the language of wave mechanics, $|L\rangle$ and $|R\rangle$ represent narrow wave packets. Note that the spatial part of $|\Phi\rangle$ is symmetric in the labels 1 and 2 , while the total state is antisymmetric as required by the antisymmetrization postulate (we are dealing with fermions).

Now, if we are to accept the factorist position that the labels 1 and 2 in Eq. (2) refer to the two EPR particles, we have to reconcile ourselves to the idea that there is neither a left nor a right electron. Indeed, the spatial states associated with both 1 and 2 are exactly the

\footnotetext{
9 This is also pointed out by Berto (2017), who goes on to argue that the needed notion of "primitive thisness" or "haecceity" can be a minimal and metaphysically relatively innocuous logical construct.
} 
same, namely $1 / 2(|L\rangle\langle L|+| R\rangle\langle R|)$, so that each of the corresponding particles would be "evenly spread out" over left and right. This means that the way the EPR case is standardly understood in foundational discussions and in experimental practice, as being about two (more or less) localized systems at a large distance from each other, is at odds with the official theoretical account, namely the factorist interpretation of the indices 1 and 2 as particle labels. ${ }^{10}$

The problem is aggravated by the observation that the strange features of factorist particles persist in the classical limit of quantum mechanics. The quantum mechanical symmetrization postulates are general and universal principles, valid in all physical situations. They are not affected by limiting procedures and approximations, whatever the exact details of these limits and approximations may be. This implies that the sameness of partial traces in all factor spaces is a generic and robust feature that survives the classical limit, so that even after taking this limit all particles still possess the same properties. But such an identity of properties does of course not apply to the particles spoken about by the classical theory resulting from the limiting procedure!

It follows that if factorism is accepted, the particles that we know from classical physics cannot correspond to their quantum namesakes. For example, in the classical limit there would be no smooth transition from quantum electrons to electrons in classical electrodynamics. That is a strange and undesirable predicament: as pointed out before, the very introduction of the particle concept in physics is motivated by our classical experience and we expect at the very least that the quantum concept approximates the traditional one in classically describable situations.

Before explaining an alternative conception of quantum particles and their states that avoids these problems, we want to discuss the attempt to salvage factorism by dropping the requirement that particles be distinguishable by their individual monadic characteristics. The idea of this proposal (Saunders 2003; 2006) is that it is sufficient for objects of the same sort in general, and identical quantum particles in particular, to be different from each other by virtue of the relations they stand in, even if it is impossible to assign individuating properties to them.

\section{Weak Discernibility}

According to classical physics it is theoretically possible to have objects that are different from each other even though they possess exactly the same properties. A famous example was proposed and discussed by Black (1952): consider two perfect spheres of exactly the same material constitution, alone in relational space (in order to exclude absolute position as a distinguishing property), at some distance from each other. By stipulation, there are no physical differences that can serve to distinguish the two spheres from each other, as the configuration is perfectly symmetrical; but still there are two spheres. This is certainly a consistent theoretical possibility. But if the individuality of Black's spheres cannot be reduced to physical differences, this would seem to signal a violation of Leibniz's Principle

\footnotetext{
${ }^{10}$ As we shall discuss in sections 5 and 6 , the rejection of factorism will make it possible to interpret the state of Eq. (2) as a representation of two localized systems. However, as we shall also see, the particular ("non-trivial") form of the superposition in (2) stands in the way of a full localized particle interpretation. This will turn out to be the background of the non-locality manifested by violations of Bell inequalities in state (2).
} 
of the Identity of Indiscernibles and of our related principle, adopted in the Introduction, that individuality in physics should be physically grounded-we appear to be forced to introduce haecceities, even in classical physics (compare the discussion in the previous section).

However, as pointed out by Saunders (2003; 2006), who takes his cue from Quine (1976), Black's spheres stand in an irreflexive physical relation to one another: a relation that an entity cannot have with respect to itself. Indeed, each sphere has a non-vanishing distance to a congruent sphere of identical composition. The irreflexivity of this relation (a sphere cannot have a non-zero distance to itself, assuming the usual Euclidean topology) makes it possible to satisfy a form of Leibniz's Principle after all: if a sphere stands in a physical relation that it cannot have to itself, it logically follows that there must be at least two spheres. At the end of the day, the spheres' numerical diversity and a minimum notion of identity thus can be physically grounded. Of course, in cases like this it remains impossible to identify the objects by means of definite physical descriptions, since any description applicable to one object applies equally well to all others - it is only the numerical diversity that can be grounded. ${ }^{11}$ Objects like Black's spheres (equal in all respects but still numerically diverse by virtue of irreflexive physical relations) are called "weakly discernible".

Now, Saunders $(2003 ; 2006)$ has suggested that identical quantum particles defined in the factorist way are different objects in the same way as Black's spheres. The simplest case to consider is that of fermions: although the mixed particle states in the different factor spaces are the same, irreflexive relations may be argued to exist between systems represented in different factor spaces, due to the antisymmetry of the total state. For example, in the singlet state $\frac{1}{\sqrt{2}}\left\{|\uparrow\rangle_{1}|\downarrow\rangle_{2}-|\downarrow\rangle_{1}|\uparrow\rangle_{2}\right\}$, the relevant irreflexive relation is "having opposite spin directions". This is because the total state is an eigenstate of the combined spin operator, with eigenvalue 0 , even though the mixed spin states in $\mathcal{H}_{1}$ and $\mathcal{H}_{2}$ are the same.

From this, Saunders $(2003 ; 2006)$ and Muller and Saunders (2008) conclude that the objecthood of factorist fermions is physically grounded. Muller and Seevinck (2009) extend this argument to bosons. These authors argue that quite generally important differences are associated with different factor spaces: operators (representing physical quantities) that belong to different factor spaces always commute, whereas this need not be the case for operators defined within one and the same factor space. In particular, momentum and position operators with different factor labels always commute, but do not when defined in the same factor space. This fact can be exploited to define irreflexive relations, which in turn can be used to argue that even bosons are weakly discernible objects.

There are several controversial points here, though (Dieks and Versteegh 2008; Dieks and Lubberdink 2011). First, an argument that weak discernibility physically grounds the numerical diversity of physical objects can only have force if the irreflexive relations that are invoked are physically relevant. In the present context this raises the question of whether mathematical relations between quantities defined in different factor spaces may be interpreted as representing relations between (candidate) physical objects-but this is precisely the issue under discussion in the debate about factorism! It follows that the irreflexivity argument by itself does not show that factor labels refer to numerically diverse

\footnotetext{
11 Leibniz's Principle therefore in this case does not bestow a physically underpinned monadic individuality marker on each object, cf. Friebe (2020).
} 
physical particles: the irreflexive relations between different factor spaces can only be used to argue for weak discernibility of physical objects if it already is assumed that the factor spaces correspond to such objects. In other words, if factorism is assumed to be right, then we may conclude that the particles represented in the different factor spaces are numerically different on physical grounds. Without the presupposition of factorism we would just be studying mathematically defined irreflexive relations between component spaces of the total Hilbert space, and could only conclude that these factor spaces are different. That conclusion, however, would be trivial.

A second point to note is that the relations considered in the weak discernibility arguments are represented by Hermitean operators, whose interpretation as physical properties can be disputed. Indeed, the standard quantum doctrine is that such operators are to be used for calculating measurement results, and that the outcomes of measurement interventions should not be mistaken for what there was before the measurement. So although it is true that spin measurements on the two wings of an EPR-Bohm experiment result in opposite outcomes, it is not clear that this is translatable into a statement about a preexisting relation between objects. Indeed, the usual interpretation of the correlations predicted by the singlet state is that these correlations testify to the holistic character of the spin system and should not be explained by an appeal to relations between spin properties that exist independent of measurement.

However, in our opinion the most important objection against quantum particles as merely weakly discernible objects is that the introduction of weak discernibility does nothing to alleviate the unnaturalness and even weirdness of factorist particles. As noted before, these supposed entities are omnipresent, find themselves all in exactly the same state and share all their physical properties. ${ }^{12}$ They are therefore extremely different from what we are used to call particles in ordinary language and classical physics. Their weak discernibility, which should make their numerical distinctness physical even without allowing any physical differences between the particles, only adds to their mysteriousness instead of making them more acceptable as particles in any ordinary sense of the term. We should surely strive for a quantum particle concept that is less extravagant; one that at least approximates the classical particle notion in an appropriate limit. In order to achieve this we must abandon factorism.

\section{Quantum Particles}

An alternative way of defining and identifying quantum particles was proposed in Lubberdink (1998), Dieks and Lubberdink (2011) and Caulton (2014). Its basic idea is to associate particles not with the labels of factor spaces, but instead with the one-particle states that occur in the total $\mathrm{N}$-particle state. In fact, this follows the motivating thought of the classical analogy of factorism: in the classical case particles are also individuated by their physical states, which in that case correlate with one-particle state spaces. The correlation between unique particle states and one-particle state spaces breaks down in quantum mechanics, and it is this feature that spells the demise of factorism. But the

\footnotetext{
12 A difference with the case of Black's spheres is that even against the backdrop of non-relational space, as standardly assumed in quantum mechanics, factorist particles share all their localization properties. Black's spheres will occupy different positions once a non-relational space, or some external reference point, e.g., an observer, is introduced (Dieks and Versteegh 2008).
} 
idea of identifying particles by states and physical properties remains eminently reasonable, also in quantum mechanics.

To see how the idea of connecting particles to unique states can be implemented, consider again the antisymmetric state

$$
\frac{1}{\sqrt{2}}\left\{|L\rangle_{1}|R\rangle_{2}-|R\rangle_{1}|L\rangle_{2}\right\},
$$

in which $|L\rangle$ and $|R\rangle$ stand for two non-overlapping wave packets at a large distance from each other-one packet located on the left, the other on the right. As we have seen, factorism tells us that this state represents two particles, both in the state $W=1 / 2\{|L\rangle\langle L|+| R\rangle\langle R|\}$, so both equally "smeared out" over left and right. However, the fact that this state is characterized by two widely separated and narrow spatial regions in which something can be detected at all, suggests rather that (3) represents a situation with one particle on the left and one on the right; the results of position measurements would confirm this interpretation. Actually, this is the interpretation that is silently adopted in the actual practice of physics (for example in EPR discussions). In order to flesh out this alternative construal of state (3) the two particles should apparently be associated with the states $|L\rangle$ and $|R\rangle$, respectively, even though each of these states occurs symmetrically in both factor spaces.

In (Lubberdink 1998; Dieks and Lubberdink 2011) this idea was proposed with special attention for the case in which the one-particle states occurring in the total state do not overlap in three-dimensional space - this avoids issues relating to the non-uniqueness of the decomposition of states like (3) and forges a bridge to the classical picture, in which particles are always localized. The essential idea, however, is more general: it is to associate particles with orthogonal one-particle states instead of factor labels, and this is often possible even in situations in which the states do not have a spatial interpretation (cf. Caulton 2014). As it turns out, a general theoretical framework proposed by Ghirardi, Marinatto and Weber (2002) can be used here.

First, it has to be noted that all observables (operators representing measurable physical quantities, which in principle can be measured) of systems of identical quantum particles must be symmetric in the factor labels. That this has to be so can immediately be seen from the fact that asymmetric operators break the symmetries required by the symmetrization postulates when they operate on states, so that their use is inconsistent with these postulates. It can also be concluded from the physical consideration that interaction Hamiltonians must be symmetric because factor labels are not measurable quantities (Dieks 1990). Candidate individuating particle properties must accordingly be represented by symmetric projection operators (telling us whether or not a property is instantiated, via the eigenvalues 1 and 0 ). That means that we should not be concerned with operators of the form $P_{1} \otimes I_{2}$, with $I_{2}$ the unity operator in factor Hilbert space 2 , but rather with projection operators like

$$
P_{1} \otimes I_{2}+I_{1} \otimes P_{2}-P_{1} \otimes P_{2},
$$

with $P$ standing for the projection operator to be used in the case of a one-particle system (in which there is only one factor space). The expectation value of the operator in (4) in an (anti)symmetric state yields the probability of finding at least one particle with the 
property represented by $P$ when we perform a measurement. The last term in (4) can be left out in the case of fermions. ${ }^{13}$

The use of such symmetric projection operators makes it sometimes possible to associate a set of pure one-particle quantum states with a many-particle system even in the symmetric and antisymmetric states required by the symmetrization postulates. Indeed, such (anti)symmetric total states may be eigenstates with eigenvalue 1 of symmetric projection operators like (4), so that the probability to find the corresponding property in a measurement is 1 . When this is the case, the proposal is to associate the one-particle state on which $P$ projects with one subsystem of the many-particle system.

This procedure leads to the association of $N$ pure one-particle states with an (anti) symmetric $N$-particle state, if the following condition is satisfied: the total (anti)symmetric state can be obtained from symmetrizing or antisymmetrizing an $\mathrm{N}$-fold product state. Such (anti)symmetrized product states are all eigenvectors of symmetric projection operators of the type (4). The usefulness of this result in the present context should be clear: in the case of (anti)symmetrized product states it is possible to find a set of one-particle states that can be used for defining one-particle subsystems.

In order to successfully implement this procedure for constructing an interpretation in terms of completely distinguishable subsystems, the one-particle states that we employ for defining these subsystems should be mutually orthogonal. This orthogonality is guaranteed in the case of fermionic systems; it may, but need not, obtain in the bosonic case. In accordance with common usage and classical physics, as explained earlier, we will reserve the use of the full particle concept for subsystems that are defined by such orthogonal states- the thus defined particles are fully distinguishable by their states (they are "absolutely discernible", i.e. distinguishable by means of monadic physical properties).

Accordingly, fermionic states that are antisymmetrized product states can always be interpreted in terms of distinct particles. ${ }^{14}$ Bosonic systems that are symmetrized product states do not always admit a particle description, because the one-particle states occurring in them need not be mutually orthogonal.

How to conceptualize situations in which a particle picture is not appropriate is an important further interpretational question that deserves a separate discussion. A tentative answer is that in such cases bosons may be better described as assemblies of field quanta (in a Fock space occupation number representation-see Dieks and Versteegh (2008) and Dieks and Lubberdink (2011)). That means that in such instances the number $n$ should not be seen as the result of counting $n$ individual entities, but rather as a mass noun. This may be likened to the everyday example of a total quantity of $n$ liters of a liquid, without any well-defined subdivision of the liquid into individual liter-entities. Similarly, " $n$ bosons of the same energy, polarization, etc.," may be interpreted as an $n$-fold excited field state, rather than as a collection of individual constituents.

\footnotetext{
13 The last term of (4) was added to allow for the possibility that the same one-particle state occurs twice in the total state, which may happen in a bosonic state. Without this last term the probability would become greater than 1 in this case. In many-fermion states one-particle states cannot occur more than once.

14 Whether such general particle pictures, defined by arbitrary orthogonal one-particle states, are relevant for the explanation of experimental results depends on the kind of measurements that are performed. In measurements that do not probe the "particle basis" of orthogonal one-particle states, the particulate character of the system will not be manifest (Dieks 2020). In practice, measurement interactions will have a local character, and therefore situations in which the one-particle states are-more or less-spatially localized are most relevant for a particle interpretation, also in connection with the classical limit.
} 
This proposal for using the notion of a particle (Lubberdink 1998; Dieks and Lubberdink 2011; Caulton 2014) fits in with the actual practice of physics. The two-fermion state (3) can be used for a quick and concrete illustration. This state is an eigenstate of the symmetric projection operators $|L\rangle_{1}\left\langle\left. L\right|_{1} \otimes I_{2}+I_{1} \otimes \mid L\right\rangle_{2}\left\langle\left. L\right|_{2} \text { and } \mid R\right\rangle_{1}\left\langle\left. R\right|_{1} \otimes I_{2}+I_{1} \otimes \mid R\right\rangle_{2}\left\langle\left. R\right|_{2}\right.$, which justifies the interpretation in terms of one particle characterized by $|L\rangle$ and one particle characterized by $|R\rangle$, respectively. The state (3) thus represents one particle on the left and one on the right, even though the labels 1 and 2 are evenly distributed over $L$ and $R$. In the transition to the classical limit this has the prospect of yielding what we expect: classical particles that are localized entities, to be approximated by narrow wave packets, following approximately classical trajectories. ${ }^{15}$

So what we propose is that the identification of quantum particles should be grounded in the distinctness of physical properties, represented by one-particle projection operators and their mutually orthogonal eigenstates. ${ }^{16}$ The thus defined quantum particles can of course be labeled, on the basis of their individuating physical characteristics. Obviously, these new labels do not coincide with the factor indices occurring in the original total quantum state-the latter remain evenly distributed over all one-particle states, even in the classical limit. Our proposal is therefore squarely anti-factorist. ${ }^{17}$

\section{Non-fundamentality of the Notion of a Particle}

According to what we have discussed, fermionic states obtainable by antisymmetrizing products of one-particle states can be understood as representing particles whose individuality rests on distinctive physical properties - that is, "identical" fermions that are distinguishable. But not all fermion states possess the form of antisymmetrized product states. The EPR-Bohm state (2) already provides us with a counterexample: although its spin part

\footnotetext{
15 Narrow quantum wave packets spread out very quickly, and will therefore only be able to follow approximately classical trajectories for a very short time. For a satisfactory classical limit, and the full applicability of classical concepts, conditions must be fulfilled that counteract this dispersion, like the presence of appropriate decoherence mechanisms.

16 We should mention the important point that the decomposition in terms of such states as given in (1) is not unique. The equality of the coefficients appearing in front of the terms in the (anti)symmetric superposition is responsible for a degeneracy, so that infinitely many alternative decompositions, in addition to the one in terms of $|L\rangle$ and $|R\rangle$ are possible. So the set of properties that distinguish the quantum particles is underdetermined by the procedure we have outlined. To make the definition of the particles unique some additional ingredient is needed, which picks out a privileged particle-properties basis. One possibility is to postulate the position basis as privileged (Lubberdink 1998; Dieks and Lubberdink 2011); this ties in with the localized nature of particles in the classical limit. An alternative and more general idea is to assume that properties and states have to be defined relative to an external "observing" system, and that the interaction with this external system determines the property basis—cf. Dieks (2019) for an exploration of this idea. In many cases the latter proposal will also single out the position as privileged, because interactions are typically position-dependent. This privileged basis issue is not specific for our particle interpretation, however, but constitutes a problem area for non-collapse interpretations of quantum mechanics in general (cf. Lombardi and Dieks 2014; 2015).

17 Muller and Leegwater (2020) suggest the possibility of salvaging factorism by constructing a new tensor product Hilbert space, once anti-factorist one-particle states of the kind we have discussed have been accepted as characterizing the component particles. Their strategy is to tailor this new space so that in it the many-particle state can effectively be written as a product. However, even if this maneuver should prove mathematically and physically unproblematic, it will not rehabilitate factorism as we have defined it, namely the doctrine saying that the factor space labels in the original tensor product space, in which the symmetrization postulates apply, refer to particles.
} 
$\left\{|\uparrow\rangle_{1}|\downarrow\rangle_{2}-|\downarrow\rangle_{1}|\uparrow\rangle_{2}\right\}$ does have the form of an antisymmetrized product, the complete state displays a more complicated form of entanglement. This fact is responsible for the non-factorizability of joint probabilities of measurement outcomes on the two wings of a Bell experiment, and consequently for violations of Bell inequalities. By contrast, the following state,

$$
\frac{1}{\sqrt{2}}\left\{|L\rangle_{1}|R\rangle_{2}|\uparrow\rangle_{1}|\downarrow\rangle_{2}-|R\rangle_{1}|L\rangle_{2}|\downarrow\rangle_{1}|\uparrow\rangle_{2}\right\},
$$

does admit a particle interpretation. It results from antisymmetrizing a product state, and leads to joint probabilities for spin measurements that factorize. This means that there will be no violations of Bell inequalities and no no-go results for local models. In fact, the particle interpretation that we have outlined immediately provides a local account: the state (5) describes a situation in which there are two particles, one on the left and one on the rightthe particle on the left-hand side having spin up and the right-hand particle spin down. All results of local measurements, and the correlations between them, can in this case be accounted for as arising from interactions between the measuring device and two localized particles, each with its own spin.

The essential difference between the states (2) and (5) is that in (5) a strict correlation exists between spatial and spin states ( $L$ with $\uparrow$ and $R$ with $\downarrow$ ), which is not the case in (2). The particles represented by the state (5) can be labeled by $(L, \uparrow)$ and $(R, \downarrow)$; note again that these labels differ from the factor labels 1 and 2). We cannot similarly define co-instantiated sets of particle properties on the basis of Eq. (2).

This example illustrates two things. First, the possibility of a sensible particle interpretation of an " $N$-particle state" is not at all given a priori. In fact, most "identical particle states" will not allow a particle interpretation. This ties in with the second point illustrated by the example, namely that typical and fundamental quantum features like holism and non-locality manifest themselves exactly when a particle picture is not appropriate.

Given the pervasiveness of non-locality and holism in quantum theory, the notion of individual particles characterized by a complete set of particle properties cannot be basic on the quantum level. As we have seen, there certainly do exist quantum states that can consistently be interpreted in terms of particles-in these cases the particle picture bridges the gap between quantum and classical descriptions of the world and helps us to understand the classical limit. But in situations that are fundamentally quantum, as in the EPRBohm state and other cases of non-trivial entanglement, particle pictures are apt to mislead rather than to clarify. Consequently, the particle concept should be considered as non-fundamental: it is only when physical mechanisms come into play that wash out the quantum effects of non-trivial entanglement (decoherence must be expected to play a pivotal role here ${ }^{18}$ ), that the notion of a particle becomes applicable and fruitful. In general situations physical systems cannot be analyzed in terms of independently defined constituent parts, and in these cases a holistic view of physical systems seems appropriate.

In other words, particles describable by classical physics emerge from the quantum world when certain physical conditions relating to the breaking of global coherence are fulfilled. This case of emergence is similar to the emergence of thermodynamics from the microscopic description: new and simple regularities arise on the emergent level-but strictly speaking the "laws" representing these regularities have the status of

18 The details of the role of decoherence mechanism in this context need a separate discussion. 
approximations. Sophisticated experiments will be able to reveal the restricted validity of the emergent concepts and laws. This is true even when dealing with "particle states" like (5): this state remains a quantum state, and it is possible to design measurements that are able to bring out typical non-classical effects like interference even in this state (Dieks 2020). It is only when we refrain from "quantum probing" and stay within the realm of the standard measurement repertoire of classical physics that the classical particle description can be relied upon.

\section{Conclusion}

According to classical physics particles constitute a basic category of what there exists in the physical world. Like everyday objects, such particles are characterized by distinctive packages of properties. As we have seen, there exist situations in quantum mechanics in which a similar picture can be used, even in the case of permutation invariant identical particles states. The state given in Eq. (5) furnishes a typical example.

This possibility of giving a distinguishable particle interpretation of permutation invariant identical particle states, at least in certain cases, is very important. Without it there could be no smooth transition, in the classical limit, from quantum to classical particles. Moreover, the way in which the concept of a particle is used in experimental practice would be difficult to connect with the description given by fundamental physics without this interpretative possibility. This interpretation of permutation invariant states in terms of distinguishable particles requires the rejection of factorism, though.

In states that are not (anti)symmetrized products, like the one of Eq. (2), physical properties are not bound together in complete packages of one-particle properties. A particle picture is in such cases not adequate. ${ }^{19}$

Such more general, not-particle-like quantum states are typical of situations that manifest quantum behavior relating to non-locality, holism, and other effects of non-trivial entanglement (entanglement that cannot be reduced to the effect of the (anti)symmetrizing of product states). Experimental evidence that the world fundamentally displays such non-classical behavior - even in cases in which we would prima facie expect that a classical description is adequate-is accumulating rapidly. The classically motivated notion of a particle characterized by distinct properties does therefore not sit well with the fundamental constitution of the physical world as represented by quantum theory and as revealed in high-precision experiments. Rather, the concept of a particle should be seen as emergent: as applicable only if conditions relating to semi-classicality (primarily concerning the washing out of effects of non-trivial entanglement) are satisfied. Even then, it should be borne in mind that the particle picture has no absolute validity.

Awareness of the generally non-particulate nature of the quantum world may provide novel conceptual means for understanding quantum processes. For example, as the state of Eq. (2) shows, it is possible to have quantum states that manifest localization in individual narrow regions in space, without complete packages of one-particle properties correlated

\footnotetext{
19 One may still consider the option of thinking in terms of incomplete packages of particle properties in such situations. In the case of the state (2) this makes it possible to speak about the system on the left and the system on the right, without assigning these systems complete sets of definite particle properties. This strategy accords with the characterization of the situation often found in physical practice, in which one speaks of a left and a right particle sharing a global spin state-see the final paragraph of this section.
} 
to these individual regions: the spin part of the EPR-Bohm state does not combine with the spatial part to form a description of two individual particles-with-spin. Abandoning part of the usual particle picture in such cases, and adapting our explanatory schemes to what the formalism is suggesting to us, may well provide new conceptual instruments, for instance for comprehending quantum information transfer (Dieks 2017).

Acknowledgements We are grateful to the organizers and participants of the 2018 Bonn workshop on Individuality, Indistinguishability and (Non-)Entanglement for giving one of us (A.L.) the opportunity to present and discuss this work. We thank Tomasz Bigaj, Cord Friebe and Fred Muller for comments.

\section{Compliance with Ethical Standards}

Conflict of interest The authors declare that they have no conflict of interest.

Open Access This article is licensed under a Creative Commons Attribution 4.0 International License, which permits use, sharing, adaptation, distribution and reproduction in any medium or format, as long as you give appropriate credit to the original author(s) and the source, provide a link to the Creative Commons licence, and indicate if changes were made. The images or other third party material in this article are included in the article's Creative Commons licence, unless indicated otherwise in a credit line to the material. If material is not included in the article's Creative Commons licence and your intended use is not permitted by statutory regulation or exceeds the permitted use, you will need to obtain permission directly from the copyright holder. To view a copy of this licence, visit http://creativecommons.org/licenses/by/4.0/.

\section{References}

Berto, F. (2017). Counting the particles: Entity and identity in the philosophy of physics. Metaphysica, 18, 69-89.

Bigaj, T. (2020). How to justify the symmetrization postulate in quantum mechanics. Journal for General Philosophy of Science (in this issue).

Black, M. (1952). The identity of indiscernibles. Mind, 61, 153-164.

Bueno, O. (2014). Why identity is fundamental. American Philosophical Quarterly, 51, 325-332.

Caulton, A. (2014). Qualitative individuation in permutation-invariant quantum mechanics. arXiv :1409.0247v1 [quant-ph].

Dieks, D. (1990). Quantum statistics, identical particles and correlations. Synthese, 82, 127-155.

Dieks, D. (2017). Quantum information and locality. In O. Lombardi, et al. (Eds.), What is quantum information? (pp. 93-112). Cambridge: Cambridge University Press.

Dieks, D. (2019). Quantum mechanics and perspectivalism. In O. Lombardi, et al. (Eds.), Quantum worlds (pp. 51-70). Cambridge: Cambridge University Press.

Dieks, D. (2020). Identical quantum particles, entanglement and individuality. Entropy. https://doi. org/10.3390/e22020134.

Dieks, D., \& Lubberdink, A. (2011). How classical particles emerge from the quantum world. Foundations of Physics, 41, 1051-1064.

Dieks, D., \& Versteegh, M. A. M. (2008). Identical particles and weak discernibility. Foundations of Physics, 38, 923-934.

Ehrenfest, P., \& Ehrenfest, T. (1959). Conceptual foundations of the statistical approach in mechanics. Ithaca, NY: Cornell University Press. German original: Begriffliche Grundlagen der statistischen Auffassung in der Mechanik. Leipzig 1909: Teubner.

French, S., \& Krause, D. (2006). Identity in physics. A historical, philosophical and formal analysis. Oxford: Oxford University Press.

Friebe, C. (2020). Leibniz, Kant, and referencing in the quantum domain. Journal for General Philosophy of Science. https://doi.org/10.1007/s10838-020-09515-5.

Ghirardi, G., Marinatto, L., \& Weber, T. (2002). Entanglement and properties of composite quantum systems: A conceptual and mathematical analysis. Journal of Statistical Physics, 108, 49-122. 
Krause, D., \& Arenhart, J. R. B. (2019). Is identity really so fundamental? Foundations of Science, 24, $51-71$.

Lombardi, O., \& Dieks, D. (2014). Modal interpretations of quantum mechanics. In E. N. Zalta (Ed.), The Stanford encyclopedia of philosophy, spring (2014th ed.). Stanford: Metaphysics Research Lab of Stanford University.

Lombardi, O., \& Dieks, D. (2015). Particles in a quantum ontology of properties. In T. Bigaj \& C. Wüthrich (Eds.), Metaphysics in contemporary physics (pp. 123-144). Amsterdam/New York: Rodopi/Brill.

Lubberdink, A. (1998). De individualiseerbaarheid van identieke deeltjes (in Dutch. English title: The individualizability of identical particles). Master thesis. Utrecht: Utrecht University. http://gradthesis.andre alubberdink.nl.

Lubberdink, A. (2009). Identical particles in quantum mechanics. arXiv:0910.4642 [physics.gen-ph].

Muller, F. A., \& Leegwater, G. (2020). The case against factorism: On the labels of $\otimes$-factor Hilbert-spaces of similar particles in quantum mechanics. Journal for General Philosophy of Science. https://doi. org/10.1007/s10838-020-09514-6.

Muller, F. A., \& Saunders, S. (2008). Discerning fermions. British Journal for the Philosophy of Science, $59,499-548$.

Muller, F. A., \& Seevinck, M. (2009). Discerning elementary particles. Philosophy of Science, 76, 179-200. Quine, W. V. (1976). Grades of discriminability. Journal of Philosophy, 73, 113-116. Reprinted in Quine, W. V. (1981). Theories and things. Cambridge, MA: Harvard University Press.

Saunders, S. (2003). Physics and Leibniz's principles. In K. Brading \& E. Castellani (Eds.), Symmetries in physics: Philosophical reflections. Cambridge: Cambridge University Press.

Saunders, S. (2006). Are quantum particles objects? Analysis, 66, 52-63.

Publisher's Note Springer Nature remains neutral with regard to jurisdictional claims in published maps and institutional affiliations. 